Research Paper

\title{
Overexpression of Toll-like Receptor 4-linked Mitogen-activated Protein Kinase Signaling Contributes to Internalization of Escherichia coli in Sheep
}

\author{
Sutian Wang1* ${ }^{*}$ Yang Cao ${ }^{1 *}$, Shoulong Deng1,3, Xiaojing Jiang1, Jiahao Wang1, Xiaosheng Zhang2, Jinlong
} Zhang르, Guoshi Liu ${ }^{1}$ and Zhengxing Lian ${ }^{1 凶}$

1. Beijing Key Laboratory for Animal Genetic Improvement, National Engineering Laboratory for Animal Breeding, Key Laboratory of Animal Genetics and Breeding of the Ministry of Agriculture, College of Animal Science and Technology, China Agricultural University, Beijing, China.

2. Tianjin Institute of Animal Sciences, Tianjin, China.

3. State Key Laboratory of Stem Cell and Reproductive Biology, Institute of Zoology, Chinese Academy of Sciences, Beijing, China.

"These authors contribute equally to this work

$\bowtie$ Corresponding author: Z.L. (email: lianzhx@cau.edu.cn)

(c) Ivyspring International Publisher. This is an open access article distributed under the terms of the Creative Commons Attribution (CC BY-NC) license (https://creativecommons.org/licenses/by-nc/4.0/). See http://ivyspring.com/terms for full terms and conditions.

Received: 2018.01.31; Accepted: 2018.04.28; Published: 2018.06 .03

\begin{abstract}
Escherichia coli is one of the most common causal pathogens of mastitis in milk-producing mammals. Toll-like receptor 4 (TLR4) is important for host recognition of this bacteria. Increased activation of TLR4 can markedly enhance the internalization of $E$. coli. In this study, the relationship between TLR4 and mitogen-activated protein kinase (MAPK) signaling pathways in mediating $E$. coli internalization was evaluated in sheep monocytes. Using a TLR4-overexpressing transgenic $(\mathrm{Tg})$ sheep model, we explored the bacterial internalization mechanism in sheep. We found that monocytes of $\mathrm{Tg}$ sheep could phagocytize more bacteria and exhibited higher adhesive capacity. The specific inhibition of p38 MAPK or c-Jun N-terminal kinase (JNK) or extracellular signal-regulated kinases (ERKs) reduced TLR4-dependent internalization of bacteria into sheep monocytes. Furthermore, the inhibition of MAPK signaling down-regulated the adhesive capacity of monocytes and the expression of scavenger receptors and adhesion molecules. Taken together, the overexpression of TLR4 in transgenic sheep enhanced the internalization of $E$. coli via MAPK signaling.
\end{abstract}

Key words: Transgenic sheep, Toll-like receptor 4, internalization, MAPK

\section{Introduction}

Mastitis, defined as an inflammation of the mammary gland, is one of the most complex diseases in dairy herds. The outcome of mastitis infection depends on various factors including properties of the pathogen, environment, and host [1, 2]. Previous studies have shown that environmental pathogens like Escherichia coli (E. coli), Staphylococcus aureus, and Streptococcus are major mastitis-causing bacteria [3-5]. Phagocytosis by immunocytes is the first line of defense against bacterial infection [6]. A number of studies have indicated that toll-like receptors (TLRs) play important roles in the first line of defense against pathogens by the activation of the host innate immune response $[7,8]$. Furthermore, some TLR ligands are involved in regulating the internalization of extracellular bacteria by immunocytes $[9,10]$. To date, over 10 TLR family transmembrane proteins have been identified. TLR4 can recognize lipopolysaccharides (LPS), the major component of the cell wall of gram-negative bacteria, including E. coli. After LPS recognition by TLR4, myeloid differentiation factor 88 (MyD88) is recruited to the intracellular portion of TLR4. Then TLR4-MyD88 signaling leads to the activation of interleukin-1 receptor-associated kinase (IRAK), which functions in the activation of multiple signaling pathways including nuclear factor kappa B (NF- $\kappa \mathrm{B})$ and mitogen-activated protein kinase (MAPKs) [ p38, c-Jun N-terminal kinase (JNK) 
and extracellular signal-regulated kinases (ERKs)] signaling pathways. MAPK signaling pathways not only induce the production of pro-inflammatory cytokines (e.g., TNF-a, IL-1 $\beta$ ) but are also involved in the internalization of bacteria [11, 12]. Several recent studies have reported that $E$. coli-stimulation results in the TLR4-dependent activation of MAPK signaling in immunocytes [13, 14]. Some research groups have shown that only p38, and not JNK or ERKs, participates in TLR4-induced internalization of bacteria [12], but others have shown that p38, JNK, and ERK signaling are all involved in this process [15]. These reports have revealed that MAPK signaling is involved in bacterial internalization. Most previous studies have focused on mouse immunocytes, which are not a perfect mastitis model; however, little is known about the mechanism of bacterial internalization in sheep, which are commonly affected by mastitis.

Actin cytoskeleton re-arrangement and bacterial adhesion are essential procedures in the internalization of bacteria [16-18]. The actin cytoskeleton is important for a variety of cell functions, including migration, adhesion, phagocytosis, and apoptosis [19]. Actin polymerization is essential for phagocytosis [20, 21]. Inhibition of actin polymerization leads to a reduction in TLR-enhanced bacterial internalization [12]. A recent study has shown that the activation of MAPK signaling pathways can induce actin stress fiber formation [15]. These observations suggest that MAPK signaling influences actin cytoskeleton re-arrangement. Scavenger receptors also participate in the innate immune response, which have various ligands such as low density lipoprotein (LDL) and LPS [22-24]. Scavenger receptors exert a variety of functions, including the phagocytosis of apoptotic cells and regulation of the adhesive capacity of phagocytes [25, 26].Notably, the activation of macrophages can up-regulate SRA-dependent adhesion [27]. In addition, a recent study has shown that CD36, a class $B$ scavenger receptor, functions as a signaling molecule that can transmit signals via MAPK [28]. Furthermore, CD36 is also involved in actin polymerization [29]. Inhibition of MAPK signaling pathways reduces scavenger receptor-mediated internalization [30]. These results suggest that interactions among TLR4, MAPKs, and scavenger receptors are involved in bacterial internalization.

Our previous studies have shown that the immunocytes of TLR4-overexpressing transgenic sheep could trigger a more intense immune response to LPS stimulation, including the secretion of various interleukins, rapid neutrophil infiltration, and enhanced oxidative stress [31-33]. In our current study, we further surveyed whether the immunocytes of transgenic sheep influence bacterial internalization.

\section{Materials and Methods}

\section{Production and screening of transgenic sheep overexpressing TLR4}

Transgenic sheep were produced by microinjecting linearized vector to fertilized eggs (Fig 1A), then embryo were transplanted into recipient oviducts within $30 \mathrm{~min}$. Genetically modified sheep were identified by Southern blot. The probe used in Southern blotting was generated by PCR using the following primers: forward, 5'-ACTGGTAAAGAACT TGGAGGAGG-3'; reverse, 5'-CCTTCACAGCATTCA ACAGACC-3'. The expression of TLR4 was quantified by qRT-PCR and immunofluorescence. Sheep blood samples were collected from the jugular veins of 6 Southern blot-positive transgenic sheep and 6 wild-type sheep. Peripheral blood mononuclear cells (PBMCs) were isolated with lymphocyte separation medium (Sigma-Aldrich, St. Louis, MO, USA). TLR4 and GAPDH primers were designed (TLR4: F, 5'-ATCATCAGCGTGTCGGTTGTCA-3' and R, 5'-GC AGCCAGCAAGAAGCATCAG-3'. GAPDH: F, 5'-GC AAGTTCCACGGCACAG-3' and R, 5'-GGTTCACGC CCATCACAA-3'). Real-time PCR reactions were performed using a MX3000P instrument (Agilent Technologies, Santa Clara, CA, USA).

\section{Cells and culture conditions}

PBMCs were obtained from the peripheral blood of six transgenic sheep and six non-transgenic sheep using lymphocyte separation medium. Cells were seeded at a density of $1 \times 10^{5}$ each well; for each sample, at least three replicates were included. After incubation for $2 \mathrm{~h}$ at $37^{\circ} \mathrm{C}$ in a $5 \% \mathrm{CO}_{2}$ incubator, the non-adherent cells were removed by washing three times with phosphate-buffered saline (PBS). RPMI1640 (Gibco, Grand Island, NY, USA) medium containing 10\% FBS (Gibco) was changed every 24 hours, and the cells were incubated at $37^{\circ} \mathrm{C}$ in a $5 \%$ $\mathrm{CO}_{2}$ incubator for $48 \mathrm{~h}$.

\section{Measurements of internalization-associated genes by real-time PCR}

Total RNA from monocytes were extracted using TRIzol Reagent (Invitrogen) and cDNA was synthesized by a PrimeScript RT reagent Kit (TAKARA). The expression of TLR4, MyD88, NF- $\kappa B$, CD14, ICAM-1 and scavenger receptors mRNA was measured by real-time PCR. The primers used in this experiment are shown in Supplementary Table 1. Real-time PCR reactions were performed using a MX3000P PCR machine (Agilent Technologies, Santa Clara, CA, USA). The SYBR Premix Ex Taq II kit 
(TAKARA) was used for qRT-PCR. The data were analyzed using the comparative $2^{-\Delta \Delta C \mathrm{CT}}$ method.

\section{Transfection of siRNA for TLR4}

For silencing of TLR4 expression, sheep monocytes were transfected with siRNA-specific TLR4 (Genepharma), si-TLR4-317: sense, 5'-CCUUGA UACUGACGGGAAATT-3'; antisense, 5'-UUUCCCG UCAGUAUCAAGGTT-3'. Meanwhile, cells are treated with negative control. Cells treated with si-TLR4 and NC using Lipofectamine RNAiMAX (Invitrogen) according to the instruction. The TLR4 knock-down efficiency is detected after $48 \mathrm{~h}$ by western blot.

\section{Western blotting and ELISA analysis}

Western blotting and ELISA analysis were performed as follow. For Western blotting, equal amounts of protein of sheep monocytes were resolved on $10 \%$ SDS-polyacrylamide gel (SDS-PAGE) and transferred to polyvinylidine difluoride membrane (PVDF). After incubation with antibodies, protein bands were dected using ECL chemiluminescence. The primary antibodies were Rabbit anti-TLR4 antibody (Bioss) and anti-GAPDH (Proteintech). The second antibody was HRP-conjugated goat anti rabbit IgG (Cwbiotech). Phosphorylation levels of p38, JNK and ERK were analyzed using ELISA kit (Abcam) according to the manufacturer's instructions.

\section{Bacterial internalization assay}

To detect internalization of bacteria, monocytes were infected with E.coli. Before infection, monocytes were washed three times in PBS without antibiotics and then mixed with E. coli at an MOI of 10, centrifuged at $100 \times \mathrm{g}$ for $10 \mathrm{~min}$, and incubated at $37^{\circ} \mathrm{C}$ in a $5 \% \mathrm{CO}_{2}$ incubator for $30 \mathrm{~min}$. Cells were then washed twice with PBS and incubated in RPMI1640 medium containing 10\% FBS and gentamicin $(30 \mu \mathrm{g} / \mathrm{mL})$ for $30 \mathrm{~min}$ to kill any non-internalized bacteria. To monitor the internalized bacteria, cells were washed three times with PBS and then lysed with $1 \%$ Triton X-100. After serial dilution, the last dilution was spread onto agar plates. After overnight culture (12h) of the bacteria, the number of viable CFUs was recorded to estimate the number of internalized bacteria.

To determine whether MAPK signaling participates in bacterial internalization, p38, JNK, and ERK $1 / 2$ were inhibited using SB203580 (5 $\mu \mathrm{m})$, SP600125 $(5 \mu \mathrm{m})$ and PD98059 $(10 \mu \mathrm{m})$, respectively (Sigma-Aldrich) prior to infection with $E$. coli to inhibit their respective signaling. Bacterial internalization-associated genes involved in these signaling pathways were evaluated by qRT-PCR as described above. Primer sequences are shown in Supplementary Table 1.

\section{Immunofluorescence staining}

Monocytes were seed in 48-well plates (Corning) in each well at a density of $1 \times 10^{5}$ cell/well. After monocytes were treated with $E$. coli, four inhibitors CLI095, SB203580, SP600125, and PD98059 were used to incubate with cells for indicated time periods. Then Cells were fixed with $4 \%$ paraformaldehyde for $20 \mathrm{~min}$ at $22^{\circ} \mathrm{C}$, permeabilized with $0.1 \%$ Triton $\mathrm{X}-100$ for $5 \mathrm{~min}$ at $22^{\circ} \mathrm{C}$, and then washed twice with warm PBS $\left(37^{\circ} \mathrm{C}\right)$ for $10 \mathrm{~min}$. After a 20 min incubation with immunol staining blocking buffer (Beyotime, Shanghai, China), cells were continuously incubated with fluorescein isothiocyanate (FITC) labelled phalloidin (Sigma-Aldrich) for $1 \mathrm{~h}$ at $37^{\circ} \mathrm{C}$ in an incubator. Cells were washed three times with PBS, and F-actin content was quantified with ImageXpress (Molecular Devices).

\section{Bacterial adhesion assay}

The adhesive capacity of the monocytes was assessed by incubation with rhodamine B-labelled $E$. coli (10:1) for $15 \mathrm{~min}$ at $37^{\circ} \mathrm{C}$ and then washing three times to remove non-adhesive bacteria. Cells were then fixed using paraformaldehyde $(4 \%)$ and then subjected to ImageXpress Micro analysis.

\section{Image Acquisition on the ImageXpress}

ImageXpress micro (Molecular Devices) was used for automated imaging. In the MetaXpress software, under Acquisition Loops, laser-based focusing was enabled. Under Wavelengths, FITC, DAPI and Texas red were selected with an exposure of $500 \mathrm{~ms}$. The autofocus was set to Laser with Z-offset. The application settings for identification of mean fluorescence intensity of each cell were as follows: approximate min width, $8 \mu \mathrm{m}$; approximate

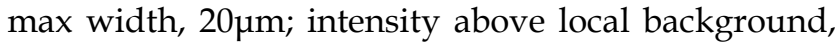
20 gray levels.

\section{Statistical analyses}

All experiments were repeated three times and the number of sheep in each group was no less than three. All data are reported as the mean \pm SEM, and all statistical analyses were performed using the Student's t-test. $\mathrm{P}<0.05$ was considered to be significant.

\section{Results}

\section{Screening of transgenic sheep}

The linear fragment containing the sheep TLR4 was microinjected into the pronuclei of fertilized eggs (Fig 1A). Founder sheep were bred with 
non-transgenic sheep (NTg). F1-generation sheep were identified by Southern blotting. After digestion of the sheep genome using Hind III, the $5-\mathrm{kb}$ band represented endogenous TLR4 on chromosome 2 , and the $3-\mathrm{kb}$ band represented the exogenous TLR4 fragment, which was only present in transgenic individuals (Fig 1B). The expression of TLR4 in transgenic-sheep monocytes derived from peripheral blood was higher than that in wild-type sheep $(\mathrm{P}<$ 0.05) (Fig $1 \mathrm{C}$ to $\mathrm{E}$ ). These findings indicated that TLR4-overexpressing sheep were successfully obtained.

\section{Overexpression of TLR4 promotes activation of MAPK signaling pathways by LPS in sheep monocytes}

First, we examined the signaling pathways activated by LPS by stimulating sheep monocytes with LPS (100 ng/mL). Compared to the NTg group, the $\mathrm{Tg}$ group showed significantly higher expression of TLR4, Myd88 and NF- $\kappa B$ after LPS stimulation at all time points $(\mathrm{P}<0.05)$ (Fig $2 \mathrm{~A}-\mathrm{C})$. Myd88 plays an important role in cell signaling transduction. Moreover, after stimulation with LPS for $30 \mathrm{~min}$, the activation of TLR4 downstream signaling, including P38, JNK, and ERK signaling, was significantly higher in the Tg group than in the NTg group $(\mathrm{P}<0.05)$ (Fig 2D-F). These results showed that TLR4 overexpression could efficiently activate $p 38, J N K$, and ERK signaling.

\section{TLR4 overexpression increases internalization of $E$. coli}

To understand the TLR4-mediated phagocytic ability of monocytes, cells of the Tg group and the NTg group were infected with E. coli at a multiplicity of infection (MOI) of 10. Then, the cells were lysed and plated on agar dishes at various dilutions for $12 \mathrm{~h}$. The intracellular bacterial counts were markedly higher in the Tg group than in the NTg group $(\mathrm{P}<$

A

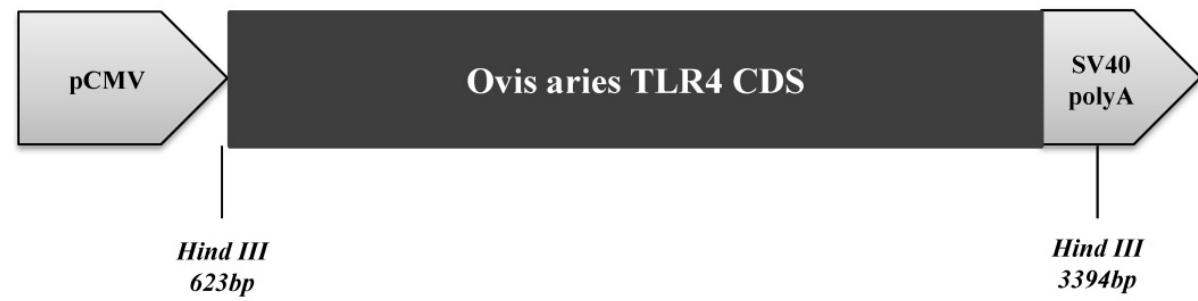

B

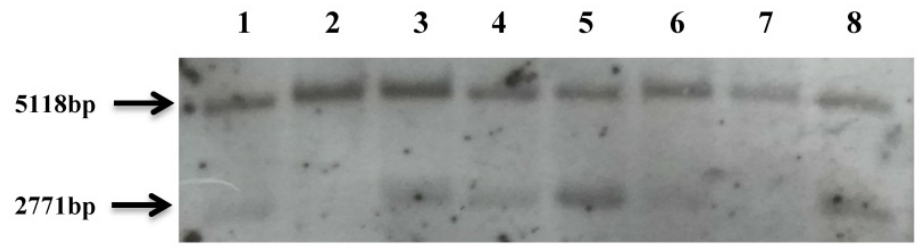

D

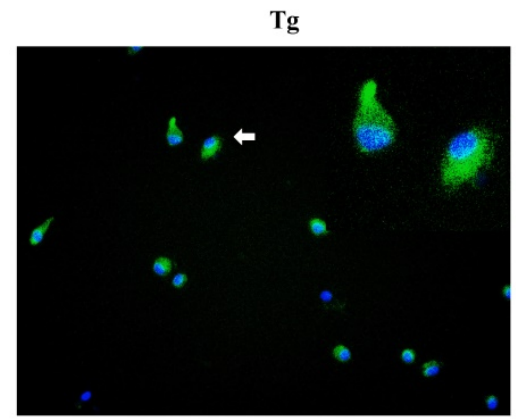

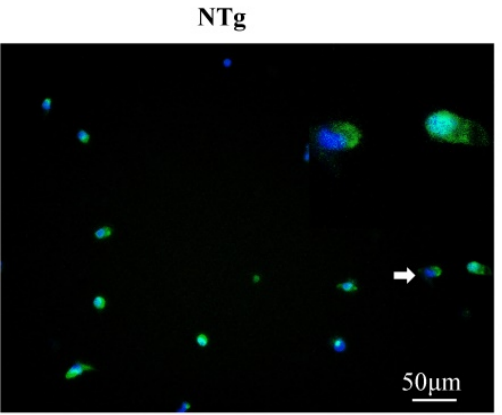

C

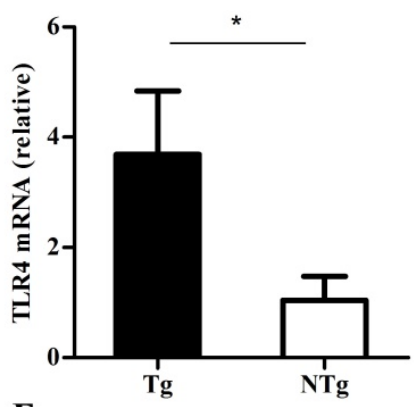

E

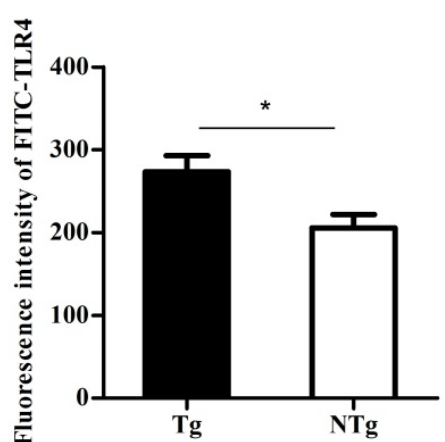

Figure 1. Screening of transgenic sheep. (A) The expression vector of ovis TLR4, which had promoter CMV and SV40 polyA. (B) Southern blot analysis of HindIII-digested sheep genomic DNA. The exogenous TLR4 fragment is the 2771 bp band, and the 5118 bp band is the endogenous TLR4 fragment. The transgenic sheep were: $1,3,4,5,8$ and the non-transgenic sheep were: 2,6 , 7. (C-E) TLR4 expression in monocytes was measured by quantitative real-time PCR (qRT-PCR) and immunofluorescence. TLR4 was labelled by FITC, nuclei were stained by Hoechst 33342 (20× ELWD ADM). Tg: transgenic sheep; NTg: non-transgenic sheep. All data are presented as the mean \pm SEM from three experiments, $* \mathrm{P}<0.05$. 
A

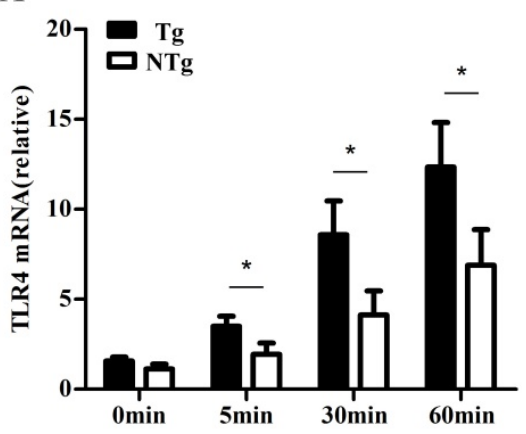

D

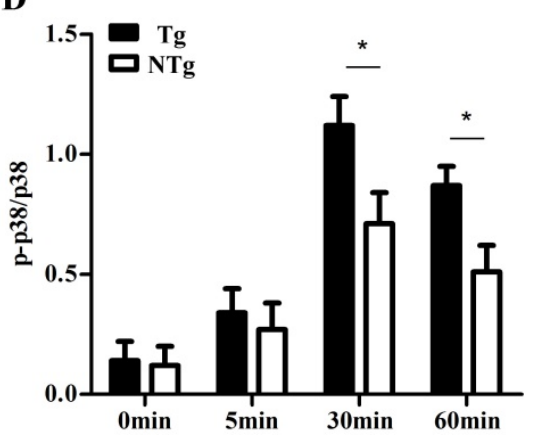

B

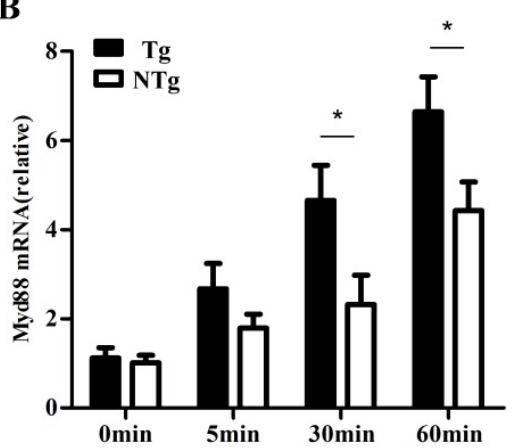

E

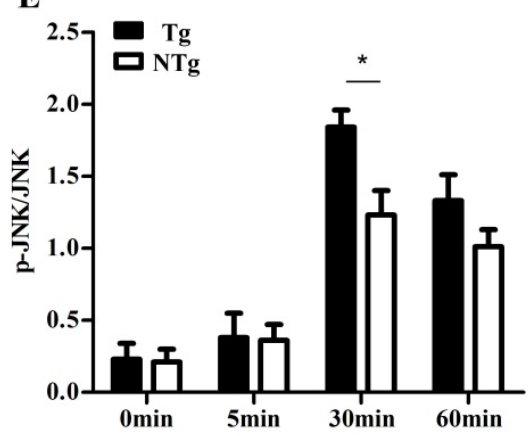

C

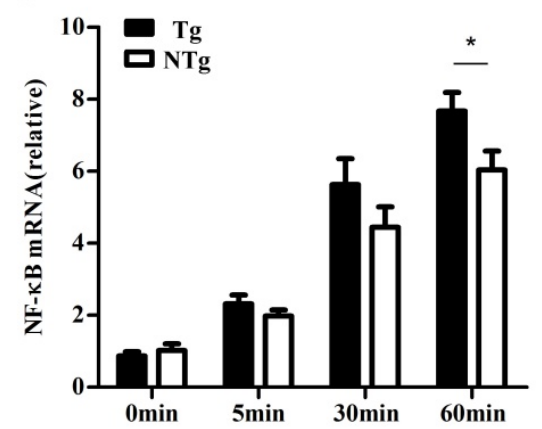

F

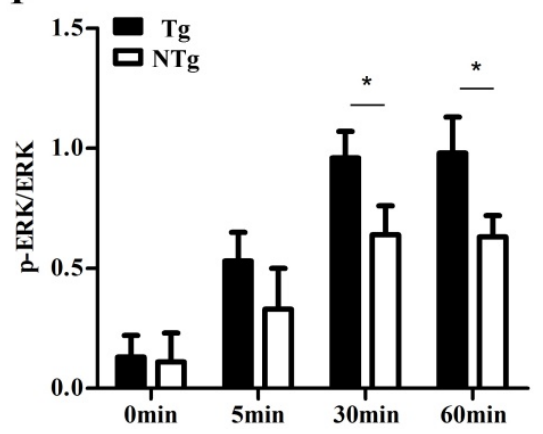

Figure 2. qRT-PCR and ELISA testing of expression in sheep monocytes with LPS stimulation. A, B and C show the expression of TLR4, Myd88 and NF- $\kappa B$, at 0 min, 5 min, 30 min and $60 \mathrm{~min}$ after LPS $(100 \mathrm{ng} / \mathrm{mL})$ stimulation, respectively. D, E and $\mathrm{F}$ show the phosphorylation levels of $p 38$, JNK, and ERK signaling at $0 \mathrm{~min}, 5 \mathrm{~min}, 30 \mathrm{~min}$ and $60 \mathrm{~min}$ after LPS $(100 \mathrm{ng} / \mathrm{mL})$ stimulation, respectively. Tg: transgenic sheep; NTg: non-transgenic sheep. All data are presented as the mean \pm SEM from three experiments, *P < 0.05 .

0.05) (Fig 3A). To determine which TLR4 downstream signaling pathways are involved in this process, we examined the transcription of multiple factors including TLR4, Myd88, and NF- $K B$ and the phosphorylation levels of $p 38, J N K$ and ERK at $30 \mathrm{~min}$ post-infection. As expected, TLR4 mRNA levels were significantly higher in the Tg group than in the NTg group $(\mathrm{P}<0.05)$ (Fig 3B). Furthermore, the levels of $M y d 88, N F-\kappa B, p 38, J N K$ and ERK were higher in the $\mathrm{Tg}$ group than in the NTg group $(\mathrm{P}<0.05)$ (Fig $3 \mathrm{C}-\mathrm{E})$. Next, we evaluated whether internalization-related receptors and genes were involved in this process. The mRNA levels of scavenger receptors and intercellular adhesion molecule 1 (ICAM-1) were influenced by the overexpression of TLR4 at $30 \mathrm{~min}$ post infection with an MOI of $10(\mathrm{P}<0.01)$ (Fig 3B). In addition, we measured the F-actin content to determine whether infection with $E$. coli affected the polymerization of actin. TLR4 overexpression enhanced the mean fluorescence intensity of F-actin of each cell population at 30 min post infection $(\mathrm{P}<0.05)$ (Fig $3 \mathrm{~F}$ and $\mathrm{G}$ ). This finding indicates that TLR4 regulates the accumulation of F-actin. Furthermore, we found that TLR4-overexpression upregulated the adhesive capacity of sheep monocytes (Fig $3 \mathrm{H}$ ). To further investigate the role of TLR4 in bacterial internalization, we inhibited the expression of TLR4 in monocytes by RNAi. Transfection of cells with si-TLR4 significantly $(\mathrm{P}<0.01)$ reduced the expression of TLR4 (Figure 3I). In addition, compared with the negative control treatment, the knock-down of TLR4 attenuated bacterial internalization (Figure 3J).

\section{TLR4-associated signaling pathways are required for internalization of $E$. coli into sheep monocytes}

Our results indicated that bacterial internalization was greater in the monocytes of the $\mathrm{Tg}$ group than in those of the NTg group; we further examined if the change was caused by the differential expression of TLR4. The number of internalized bacteria was reduced after cells were pretreated with a specific TLR4 inhibitor, CLI-095 $(10 \mu \mathrm{M})(\mathrm{P}<0.05)$ (Fig 4A). Because the specific TLR4 inhibitor could suppress the internalization of bacteria, we examined whether it could influence the rearrangement of actin cytoskeleton. As shown in Fig 4B, there was a decrease in the total amount of F-actin after E. coli infection, and this was blocked by the presence of 10 $\mu \mathrm{M}$ CLI-095. Furthermore, inhibition of TLR4 signaling reduced the adhesive capacity of sheep monocytes $(\mathrm{P}<0.05)$ (Fig 4C). To assess the effect of TLR4 on the internalization of E. coli, we detected the expression of internalization-associated genes. As shown in Fig 4D-G, CLI-095 $(10 \mu \mathrm{M})$ down-regulated multiple factors by reducing the expression of Myd88 and scavenger receptors as well as phosphorylation levels of $p 38, J N K$, and ERK $(\mathrm{P}<0.05)$. In addition, the 
mRNA levels of these genes in the $\mathrm{Tg}$ group were higher than those in the NTg group $(\mathrm{P}<0.05)$ (Fig $4 \mathrm{H})$. Our results indicate that TLR4 regulates the internalization of $E$. coli in sheep monocytes.

\section{TLR4-induced bacterial internalization is MAPK pathway-dependent}

The phagocytosis of bacteria is an important innate immune response that is mediated by monocytes. Adhesion of bacteria and actin cytoskeleton re-arrangement are essential in this process. The expression levels of $p 38, J N K, E R K$, and scavenger receptors were consistent with the internalization of bacteria, suggesting their involvement in the internalization process. To investigate if the activation of MAPK signaling plays a key role in the internalization of bacteria, sheep monocytes were pretreated with various inhibitors to block these signaling pathways and internalization activity was assessed. As shown in Fig 5A, the p38-specific inhibitor SB203580 (5 $\mu \mathrm{M})$, the JNKspecific inhibitor SP600125 $(5 \mu \mathrm{M})$ and the ERKspecific inhibitor PD98059 $(10 \mu \mathrm{M})$ all reduced the internalization of $E$. coli into monocytes. Moreover, bacterial internalization was lower in the SB203580-pretreatment group than in the other two
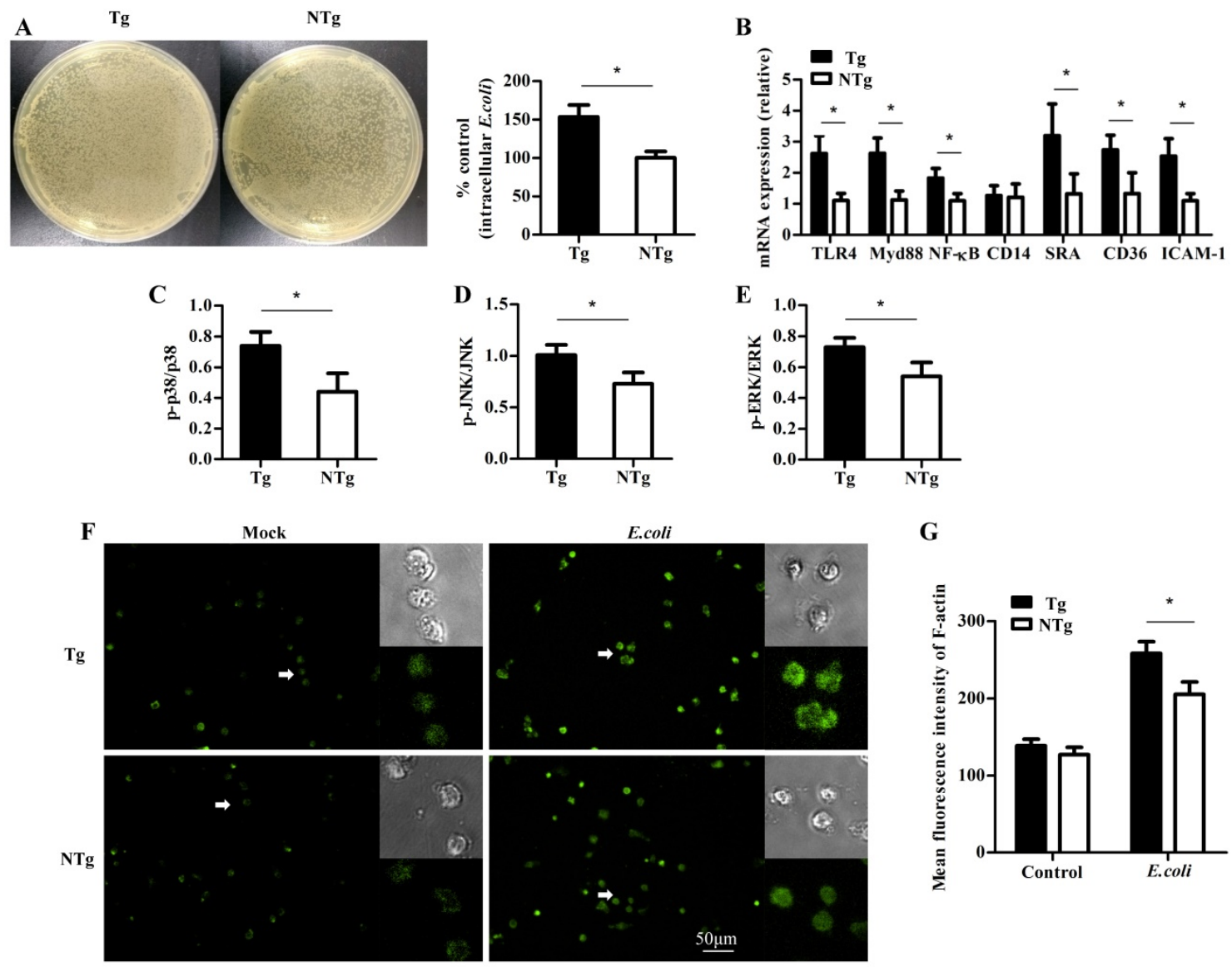

G
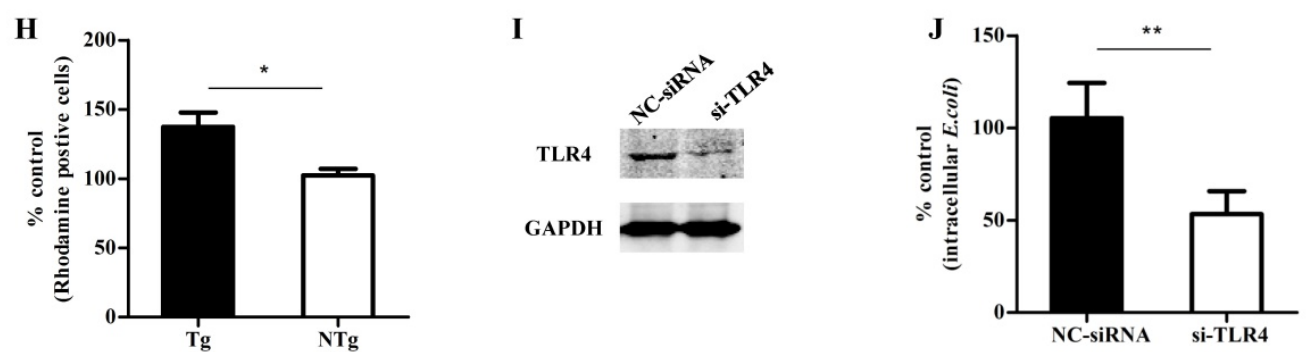

Figure 3. TLR4-mediated multiple signaling promotes internalization of $E$. coli into sheep monocytes. (A) Bacterial internalization was detected in monocytes after infection with E. coli (30 min, MOI = 10). (B) Expression of TLR4, Myd88, NF- $K B, C D I 4, S R A, C D 36$ and ICAM- $I$ after incubation with E. coli ( 30 min, MOI = 10$)$. (C to E) Phosphorylation levels of $p 38, J N K$, and $E R K$ signaling after incubation with $E$. coli $(30 \mathrm{~min}, \mathrm{MOI}=10)$. ( $\mathrm{F}$ and $\mathrm{G})$ Mean fluorescence intensity of $\mathrm{F}$-actin in each cell population; $\mathrm{F}$-actin was labelled by FITC-phalloidin (20× ELWD ADM). (H) Adhesive capacity of monocytes, E. coli was labelled by rhodamine. (I) Monocytes were transfected with negative control or TLR4 siRNA, and the expression level of TLR4 proteins was detected by western blotting. The amount of GAPDH was used as a control. (J) Negative control siRNA or TLR siRNA-transfected cells were pretreated with $E$. coli and then detected intracellular bacteria. Tg: transgenic sheep; NTg: non-transgenic sheep. All data are presented as the mean \pm SEM from three experiments, with $* \mathrm{P}<0.05$ and $* * P<0.01$. 
A

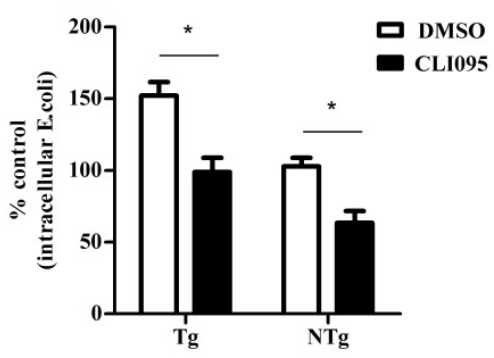

D

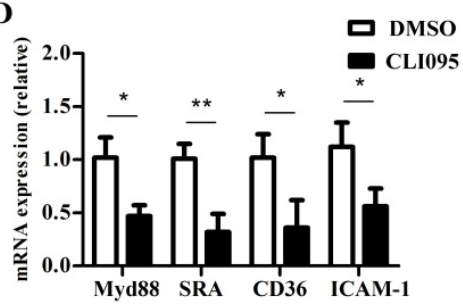

G

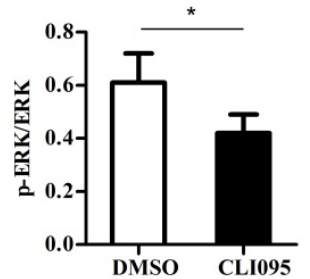

B

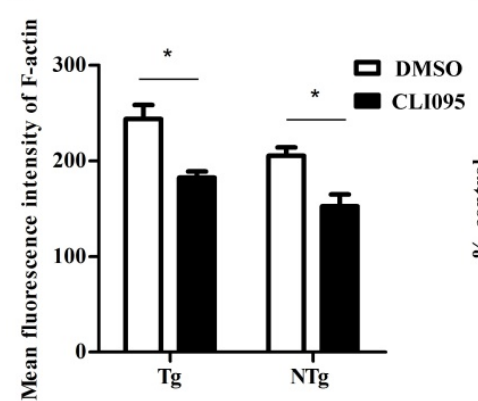

E

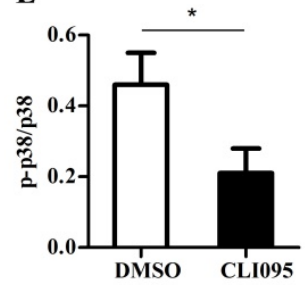

C

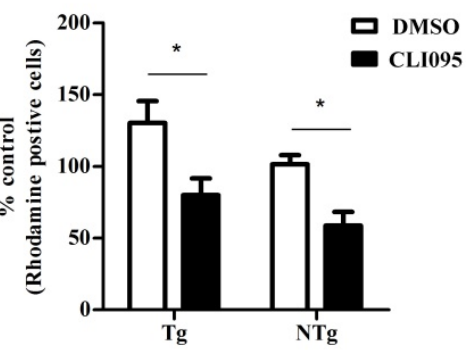

F

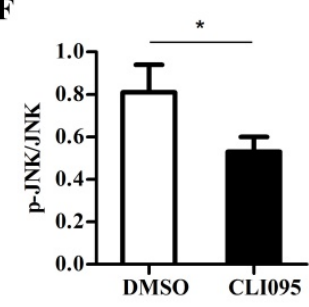

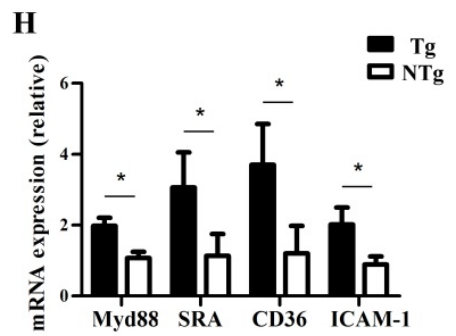

Figure 4. TLR4-associated signaling pathways are required for internalization of $E$. coli into sheep monocytes. (A) Monocytes were pretreated with DMSO (0.1\%) or CLI-095 (30 $\mu \mathrm{M})$ for $6 \mathrm{~h}$, and then bacterial internalization was detected after incubation with $E$. coli for $30 \mathrm{~min}(\mathrm{MOI}=10)$. (B) Mean fluorescence intensity of $\mathrm{F}$-actin in each cell population; F-actin was labelled by FITC-phalloidin $(30 \mathrm{~min}, \mathrm{MOI}=10)$. (C) Adhesive capacity of monocytes, $E$. coli was labelled by rhodamine. (D) qRT-PCR analysis of expression of Myd88, $S R A, C D 36$ and ICAM-1 from sheep monocytes pretreated with CLI-095 (10 $\mu \mathrm{M})$. (E to G) Phosphorylation levels of $p 38$, JNK, and ERK signaling from sheep monocytes pretreated with CLI-095 (10 $\mu \mathrm{M})$. (H) qRT-PCR analysis of expression of Myd88, SRA, CD36 and ICAM-1 from sheep monocytes pretreated with CLI-095 and then infected with E. coli (30 min, $\mathrm{MOI}=10) \mathrm{Tg}$ : transgenic sheep; NTg: non-transgenic sheep. All data are presented as the mean $\pm \mathrm{SEM}$ from three experiments, with $* \mathrm{P}<0.05$ and $* * \mathrm{P}<0.01$.

groups. E. coli-induced actin polymerization was notably lower in sheep monocytes pretreated with any of these inhibitors (Fig 5B). As expected, the adhesive capacity of sheep monocytes decreased by inhitor treatment (Fig 5C). Meanwhile, the adhesive capacity of cells was significantly lower in the SB203580-pretreatment group. We further examined the expression of internalization-associated genes. As shown in Fig 5D, the transcription of SRA in all groups was low, and the levels were lower in the SB203580-pretreated group than in the other two groups. Only the inhibition of the p38 pathway reduced the mRNA levels of CD36 and Lox-1. In addition, the expression of ICAM- 1 in all three groups was lower than that in the DMSO-pretreated control group. These data indicate that the MAPK signaling pathway plays an important role in TLR4-dependent bacterial internalization in sheep monocytes.

\section{Discussion}

TLRs are a kind of pattern recognition receptor that can recognize various molecules known as pathogen-associated molecular patterns (PAMPs) and trigger the activation of the innate immune response [34]. Several studies have reported their function in activating the inflammatory response, including the activation of $\mathrm{NF}-\mathrm{\kappa B}$ signaling and production of various inflammatory cytokines [35]. Notably, several recent studies have shown that TLR4 is involved in bacterial internalization via its interaction with MAPK pathways, which subsequently facilitate immunocyte recognition and internalization of all kinds of bacteria $[11,36,37]$. Using TLR4-overexpressing transgenic sheep, we found that the MAPK signaling pathways were associated with TLR4-dependent internalization of E. coli into sheep monocytes by promoting actin cytoskeleton re-arrangement, up-regulating the expression of multiple adhesion-related genes, and increasing the adhesive capacity of monocytes. Moreover, the knockdown of TLR4 expression by RNAi attenuated bacterial internalization. These results suggest that TLR4 has a crucial role in bacterial internalization. 

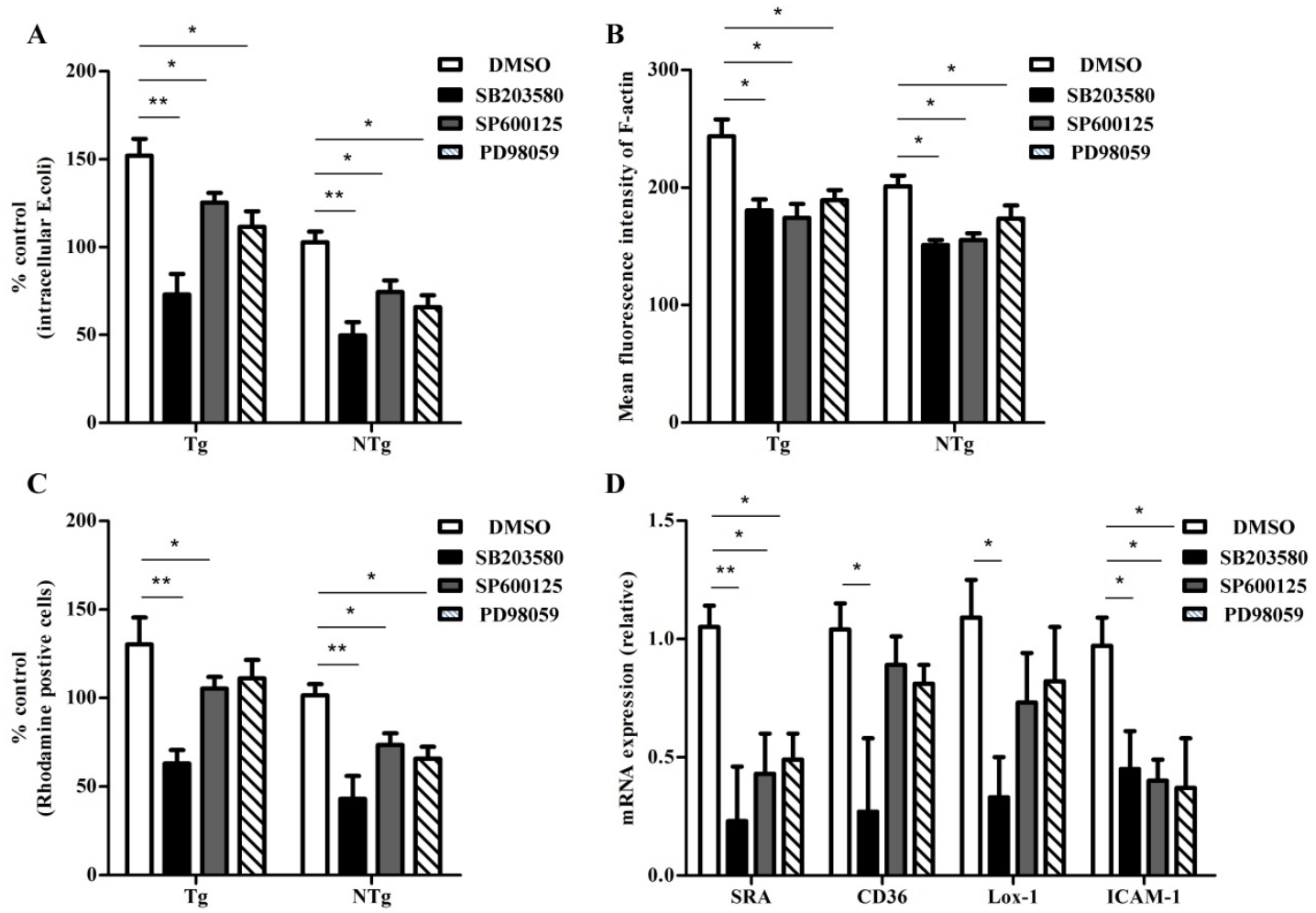

Figure 5. TLR4-induced bacterial internalization is MAPK pathway dependent in sheep monocytes. (A) Monocytes were pretreated with DMSO (0.1\%), SB203580 (5 $\mu$ M), SP600125 (5 $\mu \mathrm{M})$, and PD98059 $(10 \mu \mathrm{M})$ for 1 hour and then bacterial internalization was detected after incubation with $E$. coli for 30 min $(\mathrm{MOI}=10)$. (B) Mean fluorescence intensity of F-actin of each cell population. (C) Adhesive capacity of monocytes was detected after pretreated with DMSO (0.1\%), SB203580 (5 $\mu M)$, SP600125 (5 $\mu$ M), and PD98059 (10 $\mu M)$ for 1 hour. (D) qRT-PCR analysis of expression of SRA, CD36, Lox- I, and ICAM- I from sheep monocytes pretreated with DMSO (0.1\%), SB203580 (5 $\mu M)$, SP600125 (5 $\mu \mathrm{M})$, and PD98059 $(10 \mu \mathrm{M})$ and infected with E.coli $(30 \mathrm{~min}, \mathrm{MOI}=10)$. Tg: transgenic sheep; NTg: non-transgenic sheep. All data are presented as the mean \pm SEM from three experiments, with $* \mathrm{P}<0.05$ and $* * \mathrm{P}<0.01$.

As a major component of the cell wall of E. coli and a ligand of TLR4, LPS can quickly induce the activation of various signaling pathways in only a few minutes [38]. In particular, the NF- $\mathrm{KB}$ and MAPKs pathways are involved in the activation of inflammatory responses [35]. Consistent with these observations, we previously showed that monocytes of TLR4-overexpressing transgenic sheep produced more TNF-a, IL-6, and nitric oxide (NO) and activated more intense oxidative damage in response to LPS stimulation [31]. In this study, we showed that the overexpression of TLR4 could efficiently activate LPS-induced Myd88, P38, JNK, and ERK1/2 signaling in sheep monocytes.

An interesting study indicated that after transfecting HEK-293 cells lacking TLR4 expression and incapable of bacterial phagocytosis with functional TLR4, the transfected cells acquired the capacity for phagocytosis [39]. These findings suggest that there is a relationship between TLR4 expression and bacterial internalization. In our study, after E. coli infection, the number of intracellular bacteria in the $\mathrm{Tg}$ group was larger than that in the NTg group. Stimulation by various gram-negative bacteria could rapidly trigger the activation of the Myd88, p38, JNK and ERK1/2 signaling pathways [12, 36], and the
MAPK family of genes plays an important role in regulating bacterial internalization [40-42]. In our experiment, we found that compared to non-transgenic controls, E. coli stimulation also caused more efficient activation of these signaling pathways in transgenic sheep. Phagocytosis is a highly complex process associated with various factors, including the activation of phagocytosis-related receptors and F-actin re-arrangement. Furthermore, these factors always interact with each other [43]. CD14 is well-known for its cooperation with TLR4 to bind to LPS. CD14 also acts in conjunction with scavenger receptors to participate in phagocytosis by macrophages [44, 45]. Scavenger receptors can also recognize LPS and activate TLR4-mediated signaling pathways and are involved in bacterial internalization $[46,47]$. Interestingly, some studies indicated that TLR4, scavenger receptors [47], and CD14 [48] are capable of cross-talk and synergy. Our results showed that the overexpression of TLR4 only increases the expression of scavenger receptors but not the expression of CD14. Notably, different types of bacterial internalization signaling are stimulated by various kinds of ligation, but F-actin re-arrangement is essential for all forms of particle ligand internalization [49]. Generally, F-actin re-arrangement 
can be assessed by F-actin content $[12,50]$. Previous studies have suggested that MAPKs and class B scavenger receptors can regulate actin polymerization $[11,12,29]$. Our study demonstrated that E. coli infection induced an increased F-actin content. Moreover, the $\mathrm{F}$-actin content in the $\mathrm{Tg}$ groups was higher than that in NTg control during bacterial infection. Furthermore, TLR4-overexpression enhanced the adhesive capacity of monocytes. To investigate whether TLR4 plays a role in E. coli internalization, monocytes were pretreated with a TLR4-specific inhibitor. Our data showed that the TLR4-specific inhibitor CLI-095 reduced the internalization of $E$. coli. In addition, inhibition of TLR4 decreased the polymerization of actin and expression of the scavenger receptors and also decreased the adhesive capacity of immunocytes. In conclusion, our results indicate that TLR4 regulates the internalization of $E$. coli in sheep monocytes.

MAPKs are important downstream signaling molecules of TLR4; however, some evidence suggests that only p38 participates in bacterial internalization [12], while other studies suggest that p38, JNK, and ERK are all involved in this process [51]. It is possible that different bacterial taxa and cell types lead to the activation of diverse signaling pathways. Accordingly, we inhibited p38, JNK, and ERK to investigate internalization-related signaling in sheep monocytes. The inhibition of all these signaling molecules reduced the number of internalized bacteria. Moreover, the number of internalized bacteria was smaller in the p38-specific inhibitor group than in the other two groups. In addition, both the level of actin polymerization and the adhesive capacity of cells were down-regulated after pretreatment with these MAPK inhibitors. Furthermore, there was no difference in F-actin content among the three groups, but the adhesive capacity of cells was lower in the p38-specific inhibitor group than in the other groups. Scavenger receptors often function by various mechanisms, including adhesion, phagocytosis, and signaling [52]. SRA-dependent macrophage adhesion can induce the activation of Rac and Cdc42 [53], which trigger actin cytoskeleton re-arrangement. Similarly, CD36 is required for in LDL-induced macrophage spreading and actin polymerization [29]. Interestingly, some studies have suggested that MAPK signaling pathways are required for scavenger receptor-mediated bacterial internalization $[11,30]$. In addition, the induction of ICAM-1 is involved in immunocyte phagocytosis and ROS generation [54]. Blocking MAPK signaling could inhibit the expression of ICAM-1 [55]. In our study, the inhibition of p38, JNK, and ERK all induced decreased expression of SRA and ICAM-1, and the inhibition of p38 resulted in the strongest down-regulation of SRA expression. Notably, only the inhibition of p38 impaired the expression of CD36 and Lox-1. These results show that MAPKs enable E. coli internalization by regulating the expression of scavenger receptors and an intercellular adhesion molecule, influencing actin cytoskeleton re-arrangement, and changing the adhesive capacity of sheep monocytes.

Our results also showed that during the internalization of E. coli, the expressions of TLR4, MAPKs, and scavenger receptors were all higher in TLR4-overexpressing transgenic sheep than in the non-transgenic group. In addition, the level of actin polymerization and adhesive capacity of sheep monocytes were also higher in the transgenic sheep used in this study. These results may partially explain why TLR4-overexpressing transgenic sheep monocytes have a higher capacity for internalization.

The TLR4 signaling plays a crucial role in the innate immune response. In the present study, TLR4 overexpression sheep activated the internalization pathways of $E$. coli to improve sheep mastitis resistance. The results presented in this manuscript provide additional evidence for the role of TLR4 in the clearance of bacteria by increasing the number of internalized bacteria, promoting actin polymerization and mediating the expression of internalizationrelated genes via MAPK signaling in sheep (Fig 6). Moreover, our results provide valuable insight into the bacterial internalization mechanisms in sheep.

\section{Abbreviations}

DMSO: dmethyl sulfoxide; ERK: extracellular signal-regulated kinase; F-actin: filamentous actin; FBS: fetal bovine serum; JNK: JNK N-terminal kinase; LDL: low density lipoprotein; LPS: lipopolysaccharide; MAPK: mitogen-activated protein kinase; NF-kb: nuclear factor kappa B; PAMP: pathogenassociated molecular pattern; PBS: pathogen associated molecular pattern; Tg: transgenic; TLR: toll-like receptor; TNF: tumour necrosis factor.

\section{Supplementary Material}

Supplementary table $\mathrm{S} 1$.

http://www.ijbs.com/v14p1022s1.pdf

\section{Acknowledgements}

This work was supported by grants from the National Transgenic Creature Breeding Grand Project (2014ZX08008-005).

\section{Ethics Committee Approval and Patient Consent}

All transgenic sheep production and sample collection procedures were performed in accordance 


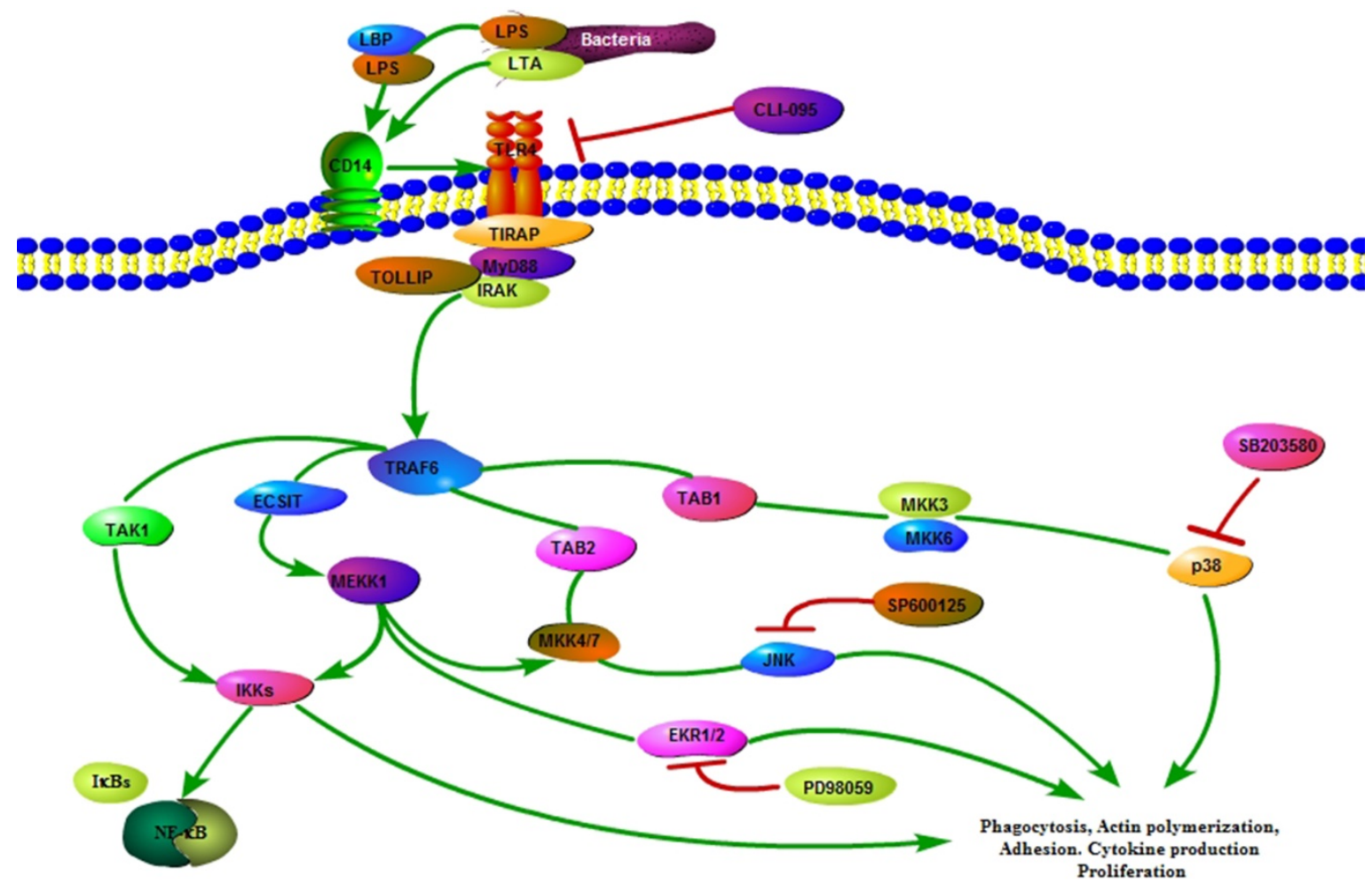

Figure 6. Diagram illustrating the hypothesized signaling pathways involved in TLR4-MAPK activation during internalization of E.coli in sheep monocytes.

with a protocol from the Animal Welfare Committee of the China Agricultural University (Permit Number: XK662).

\section{Authorship}

Sutian Wang and Zhengxing Lian designed the experiments; Sutian Wang, Yang Cao, Xiaojing Jiang, Shoulong Deng, Guoshi Liu, Xiaosheng Zhang, Jinlong Zhang, performed the experiments; Sutian Wang drafted the manuscript.

\section{Competing Interests}

The authors have declared that no competing interest exists.

\section{References}

1. De Vliegher S, Fox LK, Piepers S, McDougall S, Barkema HW. Invited review: Mastitis in dairy heifers: nature of the disease, potential impact, prevention, and control. Journal of dairy science. 2012; 95: 1025-40.

2. Schukken YH, Gunther J, Fitzpatrick J, Fontaine MC, Goetze L, Holst O, et al. Host-response patterns of intramammary infections in dairy cows. Veterinary immunology and immunopathology. 2011; 144: 270-89.

3. Rainard P, Riollet C. Innate immunity of the bovine mammary gland. Veterinary research. 2006; 37: 369-400.

4. Zadoks RN, Middleton JR, McDougall S, Katholm J, Schukken YH. Molecular epidemiology of mastitis pathogens of dairy cattle and comparative relevance to humans. Journal of mammary gland biology and neoplasia. 2011; 16: 357-72.

5. De Schepper S, De Ketelaere A, Bannerman DD, Paape MJ, Peelman L, Burvenich C. The toll-like receptor-4 (TLR-4) pathway and its possible role in the pathogenesis of Escherichia coli mastitis in dairy cattle. Veterinary research. 2008; 39: 5 .

6. Paape MJ, Schultze WD, Guidry AJ, Kortum WM, Weinland BT. Effect of an intramammary polyethylene device on the concentration of leukocytes and immunoglobulins in milk and on the leukocyte response to Escherichia coli endotoxin and challenge exposure with Staphylococcus aureus. American journal of veterinary research. 1981; 42: 774-83.
7. Akira S, Takeda K. Toll-like receptor signalling. Nature reviews Immunology. 2004; 4: 499-511.

8. Kawai T, Akira S. Pathogen recognition with Toll-like receptors. Current opinion in immunology. 2005; 17: 338-44.

9. Utaisincharoen $\mathrm{P}$, Kespichayawattana $\mathrm{W}$, Anuntagool $\mathrm{N}$, Chaisuriya $\mathrm{P}$ Pichyangkul S, Krieg AM, et al. CpG ODN enhances uptake of bacteria by mouse macrophages. Clinical and experimental immunology. 2003; 132: 70-5.

10. Dalpke AH, Schafer MK, Frey M, Zimmermann S, Tebbe J, Weihe E, et al. Immunostimulatory CpG-DNA activates murine microglia. Journal of immunology. 2002; 168: 4854-63.

11. Doyle SE, O'Connell RM, Miranda GA, Vaidya SA, Chow EK, Liu PT, et al. Toll-like receptors induce a phagocytic gene program through p38. The Journal of experimental medicine. 2004; 199: 81-90.

12. Kong L, Ge BX. MyD88-independent activation of a novel actin-Cdc42/Rac pathway is required for Toll-like receptor-stimulated phagocytosis. Cell research. 2008; 18: 745-55.

13. Xue Y, Zhang H, Wang H, Hu J, Du M, Zhu MJ. Host inflammatory response inhibits Escherichia coli O157:H7 adhesion to gut epithelium through augmentation of mucin expression. Infection and immunity. 2014; 82: 1921-30.

14. Wu Y, Ren D, Chen GY. Siglec-E Negatively Regulates the Activation of TLR4 by Controlling Its Endocytosis. Journal of immunology. 2016; 197: 3336-47.

15. Zhang L, Xu P, Wang X, Zhang M, Yan Y, Chen Y, et al. Activin B regulates adipose-derived mesenchymal stem cells to promote skin wound healing via activation of the MAPK signaling pathway. The international journal of biochemistry \& cell biology. 2017; 87: 69-76.

16. Freeman SA, Grinstein S. Phagocytosis: receptors, signal integration, and the cytoskeleton. Immunological reviews. 2014; 262: 193-215.

17. Pommier CG, Inada S, Fries LF, Takahashi T, Frank MM, Brown EJ. Plasma fibronectin enhances phagocytosis of opsonized particles by human peripheral blood monocytes. The Journal of experimental medicine. 1983; 157: 1844-54

18. Wright SD, Craigmyle LS, Silverstein SC. Fibronectin and serum amyloid P component stimulate $\mathrm{C} 3 \mathrm{~b}-$ and $\mathrm{C} 3 \mathrm{bi}$-mediated phagocytosis in cultured human monocytes. The Journal of experimental medicine. 1983; 158: 1338-43.

19. Rombouts K, Mello T, Liotta F, Galli A, Caligiuri A, Annunziato F, et al. MARCKS actin-binding capacity mediates actin filament assembly during mitosis in human hepatic stellate cells. American journal of physiology Cell physiology. 2012; 303: C357-67.

20. Caron E, Hall A. Identification of two distinct mechanisms of phagocytosis controlled by different Rho GTPases. Science. 1998; 282: 1717-21.

21. Cox D, Chang P, Zhang Q, Reddy PG, Bokoch GM, Greenberg S. Requirements for both Rac1 and Cdc42 in membrane ruffling and phagocytosis in leukocytes. The Journal of experimental medicine. 1997; 186: 1487-94.

22. Sastry K, Ezekowitz RA. Collectins: pattern recognition molecules involved in first line host defense. Current opinion in immunology. 1993; 5: 59-66. 
23. Krieger M, Herz J. Structures and functions of multiligand lipoprotein receptors: macrophage scavenger receptors and LDL receptor-related protein (LRP). Annual review of biochemistry. 1994; 63: 601-37.

24. Stahl PD ER. The mannose receptor is a pattern recognition receptor involved in host defense. Current opinion in immunology. 1998; 10: 50-5.

25. Hsu HY, Hajjar DP, Khan KM, Falcone DJ. Ligand binding to macrophage scavenger receptor-A induces urokinase-type plasminogen activator expression by a protein kinase-dependent signaling pathway. The Journal of biological chemistry. 1998; 273: 1240-6.

26. Hsu HY, Chiu SL, Wen MH, Chen KY, Hua KF. Ligands of macrophage scavenger receptor induce cytokine expression via differential modulation of protein kinase signaling pathways. The Journal of biological chemistry. 2001; 276: 28719-30.

27. van Velzen AG, Suzuki H, Kodama T, van Berkel TJ. The role of scavenger receptor class $\mathrm{A}$ in the adhesion of cells is dependent on cell type and cellular activation state. Experimental cell research. 1999; 250: 264-71.

28. Moore KJ, El Khoury J, Medeiros LA, Terada K, Geula C, Luster AD, et al. A CD36-initiated signaling cascade mediates inflammatory effects of beta-amyloid. The Journal of biological chemistry. 2002; 277: 47373-9.

29. Park YM, Febbraio M, Silverstein RL. CD36 modulates migration of mouse and human macrophages in response to oxidized LDL and may contribute to macrophage trapping in the arterial intima. J Clin Invest. 2009; 119: 136-45.

30. Sulahian TH, Imrich A, Deloid G, Winkler AR, Kobzik L. Signaling pathways required for macrophage scavenger receptor-mediated phagocytosis: analysis by scanning cytometry. Respiratory research. 2008; 9: 59 .

31. Deng S, Wu Q, Yu K, Zhang Y, Yao Y, Li W, et al. Changes in the relative inflammatory responses in sheep cells overexpressing of toll-like receptor 4 when stimulated with LPS. PloS one. 2012; 7: e47118.

32. Deng S, Yu K, Wu Q, Li Y, Zhang X, Zhang B, et al. Toll-Like Receptor 4 Reduces Oxidative Injury via Glutathione Activity in Sheep. Oxidative medicine and cellular longevity. 2016; 2016: 9151290.

33. Deng S, Yu K, Zhang B, Yao Y, Wang Z, Zhang J, et al. Toll-Like Receptor 4 Promotes NO Synthesis by Upregulating GCHI Expression under Oxidative Stress Conditions in Sheep Monocytes/Macrophages. Oxidative medicine and cellular longevity. 2015; 2015: 359315

34. Kawai T, Akira S. The role of pattern-recognition receptors in innate immunity: update on Toll-like receptors. Nature immunology. 2010; 11: 373-84.

35. Kawai T, Akira S. TLR signaling. Cell death and differentiation. 2006; 13: 816-25.

36. Kim JJLDH. Toll-Like Receptor 4-Linked Janus Kinase 2 Signaling Contributes to Internalization of Brucella abortus by Macrophages. Infection and immunity. 2013; 81: 2448-58.

37. Wong CE, Sad S, Coombes BK. Salmonella enterica serovar typhimurium exploits Toll-like receptor signaling during the host-pathogen interaction. Infection and immunity. 2009; 77: 4750-60.

38. Deng M, Scott MJ, Loughran P, Gibson G, Sodhi C, Watkins S, et al. Lipopolysaccharide clearance, bacterial clearance, and systemic inflammatory responses are regulated by cell type-specific functions of TLR4 during sepsis. Journal of immunology. 2013; 190: 5152-60.

39. Neal MD, Leaphart C, Levy R, Prince J, Billiar TR, Watkins S, et al. Enterocyte TLR4 mediates phagocytosis and translocation of bacteria across the intestinal barrier. Journal of immunology. 2006; 176: 3070-9.

40. Nick JA, Avdi NJ, Gerwins P, Johnson GL, Worthen GS. Activation of a p38 mitogen-activated protein kinase in human neutrophils by lipopolysaccharide. Journal of immunology. 1996; 156: 4867-75.

41. Schnyder B, Meunier PC, Car BD. Inhibition of kinases impairs neutrophil activation and killing of Staphylococcus aureus. The Biochemical journal. 1998; 331: 489-95.

42. Downey GP, Butler JR, Tapper H, Fialkow L, Saltiel AR, Rubin BB, et al. Importance of MEK in neutrophil microbicidal responsiveness. Journal of immunology. 1998; 160: 434-43.

43. M.Underhill AD. MECHANISMS OF PHAGOCYTOSIS IN MACROPHAGES. Annu Rev Immunol. 1999; 17: 593-623.

44. Devitt A, Moffatt OD, Raykundalia C, Capra JD, Simmons DL, Gregory CD. Human CD14 mediates recognition and phagocytosis of apoptotic cells. Nature. 1998; 392: 505-9.

45. Platt N, Suzuki H, Kurihara Y, Kodama T, Gordon S. Role for the class A macrophage scavenger receptor in the phagocytosis of apoptotic thymocytes in vitro. Proceedings of the National Academy of Sciences of the United States of America. 1996; 93: 12456-60.

46. Yu H, Ha T, Liu L, Wang X, Gao M, Kelley J, et al. Scavenger receptor A (SR-A) is required for LPS-induced TLR4 mediated NF-kappaB activation in macrophages. Biochimica et biophysica acta. 2012; 1823: 1192-8.

47. Cao $\mathrm{D}$, Luo J, Chen $\mathrm{D}, \mathrm{Xu} \mathrm{H}$, Shi $\mathrm{H}$, Jing $\mathrm{X}$, et al. CD36 regulates lipopolysaccharide-induced signaling pathways and mediates the internalization of Escherichia coli in cooperation with TLR4 in goat mammary gland epithelial cells. Scientific reports. 2016; 6: 23132.

48. Ben Haij N, Planes R, Leghmari K, Serrero M, Delobel P, Izopet J, et al. HIV-1 Tat Protein Induces Production of Proinflammatory Cytokines by Human Dendritic Cells and Monocytes/Macrophages through Engagement of TLR4-MD2-CD14 Complex and Activation of NF-kappaB Pathway. PloS one. 2015; 10: e0129425.

49. Mao Y, Finnemann SC. Regulation of phagocytosis by Rho GTPases. Small GTPases. 2015; 6: 89-99.
50. Karavitis J, Murdoch EL, Deburghgraeve C, Ramirez L, Kovacs EJ. Ethanol suppresses phagosomal adhesion maturation, Rac activation, and subsequent actin polymerization during FcgammaR-mediated phagocytosis. Cellular immunology. 2012; 274: 61-71.

51. Hop HT, Arayan LT, Reyes AWB, Huy TXN, Baek EJ, Min W, et al. Inhibitory Effect of the Ethanol Extract of a Rice Bran Mixture Comprising Angelica gigas, Cnidium officinale, Artemisia princeps, and Camellia sinensis on Brucella abortus Uptake by Professional and Nonprofessional Phagocytes. Journal of microbiology and biotechnology. 2017; 27: 1885-91.

52. PrabhuDas MR, Baldwin CL, Bollyky PL, Bowdish DME, Drickamer $\mathrm{K}$, Febbraio M, et al. A Consensus Definitive Classification of Scavenger Receptors and Their Roles in Health and Disease. Journal of immunology. 2017; 198: 3775-89.

53. Nikolic DM, Gong MC, Turk J, Post SR. Class A scavenger receptor-mediated macrophage adhesion requires coupling of calcium-independent phospholipase $\mathrm{A}(2)$ and 12/15-lipoxygenase to Rac and Cdc42 activation. The Journal of biological chemistry. 2007; 282: 33405-11.

54. Woodfin A, Beyrau M, Voisin MB, Ma B, Whiteford JR, Hordijk PL, et al. ICAM-1-expressing neutrophils exhibit enhanced effector functions in murine models of endotoxemia. Blood. 2016; 127: 898-907.

55. Liou CJ, Huang WC. Casticin inhibits interleukin-1beta-induced ICAM-1 and MUC5AC expression by blocking NF-kappaB, PI3K-Akt, and MAPK signaling in human lung epithelial cells. Oncotarget. 2017; 8: 101175-88. 